\title{
El análisis de la comunicación paraverbal cinésica y proxémica de los instructores de fitness mediante patrones temporales (T-patterns)
}

\author{
Analysis of kinesic and proxemic paraverbal communication \\ in fitness instructors using time patterns (T-patterns)
}

\section{Análise da comunicação paraverbal cinésica e proxêmica em instrutores de fitness atraves padróes temporais (T-patterns)}

\author{
Susana Alves ${ }^{1,3,}$, Susana Franco ${ }^{1,4}$, Marta Castañer $^{2}$, Oleguer Camerino ${ }^{2}$, José Rodrigues ${ }^{5,6}$ y Raúl Hileno ${ }^{2}$ \\ ${ }^{1}$ Escola Superior de Desporto de Rio Maior (ESDRM)- Instituto Politécnico de Santarém, (Portugal), \\ ${ }^{2}$ Laboratorio de Observación de la Motricidad-Instituto Nacional de Educación Física de Cataluña (INEFC)-Universidad de Lleida (España), \\ ${ }^{3}$ Centro de Investigación en Calidad de Vida (CIEQV) - Santarém (Portugal), ${ }^{4}$ Laboratório de Biociências da Motricidade Humana (LABIMH) (Brasil), \\ ${ }^{5}$ IP Santarém International School (Portugal), ${ }^{6}$ Laboratório de Biociências da Motricidade Humana (LABIMH) (Brasil)
}

\begin{abstract}
Resumen: El objetivo de este estudio es el análisis de la comunicación paraverbal y los perfiles comunicativos de los instructores de programas de fitness: step, tonificación, fitness acuático y cycling. Aplicamos la metodología observacional para grabar videográficamente doce instructoras expertas (n = 12 mujeres, edad de $31 \pm 6.14$ ańos), a razón de tres sesiones de cada uno de estos cuatro programas, que registramos con los instrumentos de observación SOCIN-fitness, SOPROX-fitness y codificado mediante el software LINCE. Los resultados descriptivos y de patrones temporales (T-patterns), obtenidos respectivamente mediante los programas SPSS v.20.0. y THEME 5.0., revelan una gran variabilidad en el comportamiento cinésico y proxémico de las instructoras de cada actividad, y también un predominio gestual condicionado por la ejecución con escasa riqueza interactiva y participativa hacia los usuarios. La optimización de los intercambios comunicativos de las instructoras es esencial para promover una mayor motivación y aceptación de los programas de fitness de la población.

Palabras claves: comunicación paraverbal, metodología observacional, Tpatterns, fitness

Abstract: The aim of this study was to analyze paraverbal communication and communication patterns in fitness instructors in four disciplines: body step, toning, aqua fitness, and indoor cycling. Using observational methodology, we filmed 12 workout sessions ( 3 for each discipline) led by twelve expert instructors (all women, with a mean age of $31 \pm 6.14$ years). The sessions were observed using the SOCIN-fitness and SOPROX-fitness observation instruments and analyzed using the LINCE multiplatform sports analysis software. Descriptive results and time patterns (T-patterns)
\end{abstract}

yielded by SPSS v.20.0 and THEME 5.0, respectively, revealed marked variability in kinesic and proxemic behaviors in all 4 disciplines, in addition to a predominance of gestures marked by the execution of the activities, with little interaction and participation with members of the class. Optimization of communication between fitness instructors and participants is key to improving motivation and acceptance of fitness programmes among the general population.

Keywords: paraverbal communication, observational methodology, Tpatterns, fitness

Resumo: O objetivo deste estudo é analisar padrốes de comunicaçáo paraverbal[comunicação não verbal $(\mathrm{CNV})]$ de uma amostra representativa dos instrutores de programas de fitness das atividades de: step, localizada, hidroginástica e cycling. Foram filmados em vídeo 3 sessóes de cada tipo de atividade de doze instrutores experientes ( $\mathrm{n}=12$ mulheres, $31 \pm 6.14$ anos de idade), e observados com os instrumentos de observação SOCINfitness e SOPROX-fitness, sendo o registo efetuado no software LINCE. Os resultados descritivos e os padrôes temporais (T-patterns), obtidos através dos programas SPSS v20.0 e THEME 5.0, respetivamente, revelaram uma grande riqueza na comunicação cinésico e gestual acompanhadas de uma grande variabilidade proxémica na orientação e posição corporal no espaço. A caracterização e deteção destes perfis de comunicação e otimização no desenvolvimento profissional pode levar a uma maior adesão a programas de fitness e aumentar a motivação dos seu praticantes.

Palavras chave: comunicaçẫo paraverbal, metodologia observacional, padrôes-T, fitness

\section{Introducción}

La práctica de las actividades físicas dirigidas por profesionales puede asegurar niveles de condición física saludable (Battista et al., 2008; Berry, Cline, Berry y Davis, 1992). La comunicación positiva que promueven las sesiones de fitness

Dirección para correspondencia [Correspodence address]: Susana Alves. Escola Superior de Desporto de Rio Maior. Av. Dr. Mário Soares. 2040 413 Rio Maior (Portugal). E-mail: salves@esdrm.ipsantarem.pt en grupo y las sensaciones de autoconfianza que promueven sus profesionales asegura el éxito de los programas, la existencia de un buen clima en las sesiones aumenta la motivación y la adhesión de sus practicantes (Moreno y Martínez, 2006; Moreno, et al. 2010).

La comunicación paraverbal (CPV) es un buen recurso para estos profesionales de las actividades físicas dirigidas ya 
que les permite vincularse con los participantes en ambientes dispersos y difíciles (Castañer, Franco, Rodrigues, y Miguel, 2012; Castañer, Camerino, Anguera, y Jonsson, 2013). El componente gestual de la comunicación y los movimientos realizados esencialmente con los brazos y manos es parte integrante del proceso comunicativo (Cartmill, Goldin-Meadow, y Beilock, 2012; Kendon, 2004) y contribuye significativamente a su eficacia según comprobó Hostetter (2011) a partir de un metaanálisis de la oportunidad de la comunicación

En los programas de fitness nos encontramos con factores que dificultan la comunicación: el volumen elevado de la música, que entorpece la instrucción verbal (Alves et al., 2014; Mirbod et al., 1994; Yarenchuk y Kaczor, 1999); la adecuación del gesto a la ejecución del movimiento (Castañer, et al. 2012; Franco, Rodrigues y Castañer, 2008), y la necesidad de mantener una proximidad espacial con los practicantes (Alves et al., 2013).

No obstante, los profesionales de estos programas de fitness, de forma específica en cada actividad (Franco y Santos, 1999), optimizan su instrucción con recursos interactivos de comunicación cinésica o gestualidad corporal y adaptan su comunicación proxémica con relación al uso del espacio (Knapp y Hall, 2010). El éxito comunicativo de los profesionales de fitness depende de una utilización eficaz de los códigos cinésico-gestuales que refuerzan las informaciones verbales e indican los cambios espaciales en la sala (Kennedy y Yoke, 2004).

El análisis de la CPV en el contexto de la enseñanza de la educación física mediante los sistemas de observación SOCIN y del SOPROX (Castañer, 2009; Castañer, Camerino, Anguera y Jonsson, 2010) nos identificaron patrones comunicativos de los enseñantes sin experiencia comparados con los enseñantes expertos. Estos sistemas SOCIN y SOPROX se utilizaron para estudiar las conductas comunicativas de entrenadores de fútbol en diferentes competiciones y han aportado patrones singulares en cada caso (Castañer et al., 2012). En esta línea de investigación el estudio de Castañer et al., (2013) comprobó que los profesores noveles, a pesar de realizar una mayor cantidad de gestos, presentan una comunicación gestual de menor calidad pero con un aprovechamiento de la ocupación del espacio que optimiza los aprendizajes de los alumnos.

El objetivo de esta investigación es identificar las conductas paraverbales cinésicas y proxémicas de una muestra de instructores cualificados de las principales actividades dirigidas de fitness en los contextos naturales de su actuación, analizando los patrones temporales (T-patterns) de su comunicación paraverbal para contribuir a su conocimiento y optimizar sus estrategias de intervención.

\section{Método}

Utilizamos la metodología observacional que es útil en el ámbito complejo de la interacción comunicativa al cumplir unos requisitos básicos: implementación en contextos naturales, elaboración de instrumentos de observación ad hoc, y continuidad temporal (Anguera, Blanco-Villaseñor, Hernández-Mendo y Losada, 2011). Esto nos permitió el registro sistemático y el análisis pormenorizado de los comportamientos comunicativos de estos profesionales que podrían ser contrastados, en otros estudios, con datos cualitativos en un enfoque Mixed Methods de la investigación de las Ciencias de la Actividad Física y el Deporte (Camerino, Castañer y Anguera, 2012).

El diseño observacional (Anguera, Blanco-Villaseñor y Losada, 2001, Anguera, Blanco-Villaseñor, HernándezMendo y Losada, 2011) es N/P/M: nomotético (centrado en el análisis comparativo de cada grupo de instructores según su especialidad), puntual (se han analizado una única sesión de cada profesor) y multidimensional (observamos varias dimensiones).

\section{Participantes}

El estudio estuvo compuesto por 12 instructoras de los cuatro programas: step, tonificación, fitness acuático y cycling, sobre los que se han definido los siguientes criterios de inclusión: (a) mujeres, para evitar la influencia del género sobre el tipo de comunicación (Kennedy y Camden, 1983); (b) ser tituladas superiores en Ciencias de la Actividad Física y el Deporte, ya que el nivel de formación pueden condicionar su actuación docente (Malek, Nalbone, Berger y Coburn, 2002); (c) un mínimo de 5 ańos de experiencia, unificando el bagaje profesional definido por Berliner (1994), y homogeneizando el desempeño de los profesionales de fitness participantes ( $\mathrm{Si}$ móes, Franco y Rodrigues, 2009); (d) una frecuencia mínima de impartición de 3 sesiones semanales para equiparar su dedicación profesional. La edad de los 12 participantes estaba situada entre los 24 y los 48 ańos $(M=31.50$ años; $D E=6.14)$, todos presentaban un bagaje profesional como instructoras de fitness con más de 6 años de experiencia y una frecuencia de dedicación semanal de 3 a 4 sesiones.

\section{Instrumentos de observación}

Adaptamos los sistemas de observación de la comunicación cinésica SOCIN y de la proxémica SOPROX (Castañer et al., 2010; 2013), creando dos nuevos instrumentos según Brewer y Jones (2002): el SOCIN-fitness (Alves et al., 2014), con 5 criterios y 21 categorías (Tabla 1); y el SOPROX-fitness (Alves et al., 2013), con 5 criterios y 23 categorías (Tabla 2). 
Tabla 1. Sistema de observación de la comunicación cinésica (SOCIN-Fitness).

\begin{tabular}{|c|c|c|c|}
\hline Criterio & Categoría & Código & Descripción \\
\hline Función (DF) & Regulador & RE & Búsqueda respuesta inmediata de los participantes o receptores. \\
\hline Gestos funcionales & Ilustrador & IL & Descripción de lo se expresa verbalmente sin respuesta inmediata. \\
\hline \multirow{9}{*}{$\begin{array}{l}\text { Morfología(DM) } \\
\text { Gestos significativos }\end{array}$} & Deíctico & DEI & Representación de lugares, objetos, partes de cuerpo o personas. \\
\hline & Emblema Técnico & EMBT & Icono específico del contexto del Fitness. \\
\hline & Emblema Social & EMBS & Icono acordado en las propias actividades. \\
\hline & Emblema Numérico & EMBN & Icono preestablecido que indica formas o cómputos numéricos. \\
\hline & Pictográfico & PIC & Movimientos de los brazos que dibujan figuras o formas en el espacio. \\
\hline & Cinetográfico & CIN & Acciones o movimientos representados en el espacio. \\
\hline & Espacial & ESP & Representación de distancias, objetos, segmentos corporales o personas \\
\hline & Rítmico & RIT & Marcas de tiempo o la velocidad de la acción o el ejercicio. \\
\hline & Batuta & BAT & Iconos que refuerzan la lógica repetitiva del discurso verbal. \\
\hline \multirow{4}{*}{$\begin{array}{l}\text { Situacional (DS) } \\
\text { Gestos de relación }\end{array}$} & Información & INF & Explicación del ejercicio con la gestualidad del instructor. \\
\hline & Feedback & FEED & Ayudar, corregir o evaluar a los participantes. \\
\hline & Interacción & INT & Refuerzo de ánimo a los participantes. \\
\hline & Organización & ORG & Disposición del material o alumnos en el espacio. \\
\hline \multirow{2}{*}{$\begin{array}{l}\text { Ejercicio (DE) } \\
\text { Nivel participación }\end{array}$} & Con Ejercicio & $\mathrm{CE}$ & El instructor está realizando ejercicio. \\
\hline & Sin Ejercicio & SE & El instructor no realiza ejercicio. \\
\hline \multirow{4}{*}{$\begin{array}{l}\text { Adaptador (DA) } \\
\text { Contactos instructor }\end{array}$} & Auto-adaptador & AA & Con partes de su cuerpo sin intención comunicativa. \\
\hline & Adaptador objetual & OB & Con objetos sin intención comunicativa. \\
\hline & Hetero-adaptador & HA & Con otras personas sin intención comunicativa. \\
\hline & Multi-adaptador & MUL & Combinación de adaptador objetual, auto-adaptador y hétero-adaptador. \\
\hline
\end{tabular}

Tabla 2. Sistema de observación de la comunicación proxémica (SOPROX-Fitness).

\begin{tabular}{lccl}
\hline Criterio & Categoría & Código & Descripción \\
\hline Grupo (DG) & Macro-grupo & MAC & A todos los participantes del grupo en su conjunto. \\
Tipo comunicación & Díada & DIA & A un solo participante. \\
& Micro-grupo & MIC & A un grupo concreto de participantes. \\
\hline Topología (DTO) & Periférica & P & El instructor se ubica por los laterales o extremos del espacio. \\
Ubicación instructor & Central & C & El instructor se sitúa en las zonas centrales del espacio. \\
\hline \multirow{2}{*}{ Interacción (DI) } & Integrada & IINT & Implicación pero sin contacto corporal. \\
Interacción instructor & Distanciada & DIS & Ausencia de lo que está sucediendo. \\
& Contacto táctil & CT & Implicación en lo que está sucediendo con contacto corporal. \\
\hline & De Frente Espejo & FE & En frente y de cara al campo de visión de los participantes. \\
De frente & FC & En frente y de espaldas al campo de visión de los participantes. \\
Localización instructor & De Frente Perfil & FP & A los laterales del campo de visión de los participantes. \\
& Detrás & AT & Detrás de los participantes y fuera de su campo de visión. \\
& En Medio & NM & Dentro del espacio que ocupan los participantes. \\
& Derecha & DIR & A la derecha de donde se ubican los participantes. \\
& Izquierda & ESQ & A la izquierda de donde se ubican los participantes. \\
\hline
\end{tabular}




\begin{tabular}{lccl}
\hline Criterio & Categoría & Código & Descripción \\
\hline & Bípeda Desplazamiento & PBD & Postura de pie pero con desplazamiento. \\
& Postura Fija Bípeda & PFB & De pie, de rodillas o en cuadrupedia sin desplazarse. \\
& Postura Sentada & PFS & Sin desplazarse aunque puede mover sus segmentos corporales. \\
Transición (DTR) & Postura Fija Dorsal & PFD & Sin desplazarse aunque puede mover sus segmentos corporales. \\
Postura instructor & Postura Fija Ventral & PFV & Echado sobre la zona ventral moviendo sus segmentos corporales. \\
& Postura Fija Lateral & PFL & Echado sobre el laterales de su cuerpo sin desplazarse con movimiento. \\
& Locomoción & LOC & Movimiento debido al ejercicio, de un lugar a otro del espacio. \\
& En soporte & SU & Apoyo en alguna estructura del espacio, objeto o persona.
\end{tabular}

\section{Instrumentos de registro}

Las grabaciones de los vídeos se realizó con una cámara digital (JVC GZ-MG21E) y su codificación mediante el software LINCE (Gabín, Camerino, Anguera y Castańer, 2012; Hernández-Mendo et al., 2014) que integra una amplia gama de funciones necesarias: codificación simultánea al visionado, cálculo de calidad de datos y obtención del registro en diferentes formatos para ser exportados a varias aplicaciones informáticas para su análisis observacional.

\section{Procedimiento}

La grabación de las imágenes se efectúo mediante la autorización y el consentimiento informado por escrito del responsable de la instalación deportiva, de las instructoras y de los participantes. Así, todos los procedimientos adoptados cumplen las recomendaciones éticas definidas por Harriss y Atkinson $(2009,2011)$ para la investigación en esta área. El registro se verificó con un análisis de la calidad de los datos (Anguera, y Hernández-Mendo, 2014) donde se estimó una concordancia interobservador aceptable del registro de dos observadores con unos valores del índice de Kappa de Cohen superiores al valor .75 y una concordancia intraobservador con los mismos resultados (Cohen, 1960).

\section{Análisis de datos}

Los registros observacionales proporcionados por LINCE se exportaron en formato (.spv) al paquete estadístico SPSS v.20.0 (IBM Corp., Armonk, NY) para el análisis estadístico descriptivo e inferencial, y en formato (.txt) al software THEME 5.0. (Magnusson, 2000, 2005) para la detección de patrones temporales (T-patterns).

Una vez determinada la distribución de frecuencias de la comunicación paraverbal, se analizó la relación entre categorías por medio del cálculo de los residuos ajustados. En las tablas de contingencia bidimensionales obtenidas sólo se aceptaron los residuos ajustados positivos que cumplían los requisitos de la aproximación normal (Bakeman y Quera, 1995) con un valor absoluto de $z \geq 3.29$ y un nivel de significación de $p<.001$.

Seguidamente, se aplicó la técnica de patrones temporales (T-patterns) para detectar relaciones secuenciales entre los comportamientos paraverbales, que se representan mediante gráficos en árbol o dendogramas y que ilustran la sucesión de estos patrones comportamentales, denominados T-patterns, compuestos por grupos de categorías que ocurren según un orden cronológico y dentro del mismo intervalo temporal crítico (Jonsson et al., 2010).

\section{Resultados}

\section{Análisis descriptivo}

Destacan, en la agregación total de los gestos cinésicos globales, las categorías (RE, DEI, INF, CE y AA), pero distinguimos algunas diferencias por actividades (Tabla 3):

a) Los reguladores (RE) son más importantes en actividades en las cuales la representación de consignas es importante: cycling y tonificación.

b) Los ilustradores (IL), tienen más prevalencia en actividades en las cuales la representación de consignas es importante: el fitness acuático y el steps.

c) Los emblemas técnicos (EMBT), tienen más importancia en las actividades en las cuales es indispensable marcar la ejecución como en el step.

d) La preeminencia de la información (INF) en todos los programas va asociada a un incremento del feedback (FEED) menos en el steps y el cycling en el que la interacción (INT) está en segundo orden.

e) La participación de los instructores sin el modelo de los ejercicios (SE), aparece en el fitness acuático debido a la colocación del instructor fuera del agua.

f) La gran preponderancia de los auto-adaptadores (AA) en todos los programas en frente de los adaptadores objetuales (OB) se ve disminuida en el cycling. 
Tabla 3. Frecuencias de ocurrencias de las categorías de (SOCIN-Fitness).

\begin{tabular}{|c|c|c|c|c|c|c|}
\hline Criterio & Categoría & Fitness acuático & Cycling & Tonificación & Step & Total \\
\hline \multirow[t]{2}{*}{ Función } & Regulación (RE) & 88.67 & 92.3 & 93.73 & 88.89 & 90.90 \\
\hline & Ilustración (IL) & 11.33 & 7.7 & 6.27 & 11.11 & 9.10 \\
\hline \multirow[t]{9}{*}{ Morfologia } & Deíctico (DEI) & 29.65 & 18.75 & 28.33 & 32.29 & 27.26 \\
\hline & EmblemaTécnico (EMBT) & 0.11 & 0.00 & 4.92 & 24.37 & 7.35 \\
\hline & Emblema Social (EMBS) & 14.28 & 11.33 & 18.08 & 22.67 & 16.59 \\
\hline & Emblem Numerico (EMBN) & 14.39 & 15.68 & 15.39 & 10.17 & 13.91 \\
\hline & Pictografico (PIC) & 1.65 & 5.82 & 0.97 & 0.31 & 2.18 \\
\hline & Cinetografico (CIN) & 24.30 & 22.08 & 22.54 & 6.29 & 18.80 \\
\hline & Espacial (ESP) & 0.77 & 3.14 & 1.18 & 0.09 & 1.30 \\
\hline & Ritmico (RIT) & 3.15 & 8.23 & 5.70 & 0.28 & 4.34 \\
\hline & Batuta (BAT) & 11.70 & 14.97 & 2.89 & 3.53 & 8.27 \\
\hline \multirow[t]{4}{*}{ Situacional } & Información (INF) & 60.40 & 76.05 & 76.60 & 94.21 & 76.82 \\
\hline & Feedback (FEED) & 23.48 & 10.25 & 13.72 & 2.33 & 12.45 \\
\hline & Interación (INT) & 13.18 & 13.26 & 7.03 & 3.46 & 9.23 \\
\hline & Organización (ORG) & 2.94 & 0.44 & 2.65 & 0.00 & 1.50 \\
\hline \multirow[t]{2}{*}{ Participación } & Con Ejercicio (CE) & 28.66 & 97.01 & 67.83 & 94.05 & 71.88 \\
\hline & Sin Ejercicio (SE) & 71.34 & 2.99 & 32.17 & 5.95 & 28.12 \\
\hline \multirow[t]{4}{*}{ Adaptador } & Auto-adaptador (AA) & 92.53 & 72.78 & 81.67 & 87.41 & 83.60 \\
\hline & Objectual $(\mathrm{OB})$ & 5.80 & 27.22 & 18.43 & 12.59 & 15.99 \\
\hline & Hetero-adaptador (HA) & 1.67 & 0.00 & 0.00 & 0.00 & 0.41 \\
\hline & Multi-adaptador (MUL) & 0.00 & 0.00 & 0.00 & 0.00 & 0.00 \\
\hline
\end{tabular}

Nota: resultados de las frecuencias expresados en porcentajes.

Sobresalen, en el cómputo de los gestos proxémicos de todas las actividades, las categorías (MAC, P, IINT, FE y PFB), y podemos destacar (Tabla 4):

a) Una superioridad de consignas hacia el gran grupo (MAC), que se remarca en el steps por la poca corrección individualizada.

b) Una distribución topológica del instructor periférica $(\mathrm{P})$ y muy poco central (C) sólo compensada en la tonificación.

c) Una interacción integrada en el grupo (IINT) frente a una escasa interacción distanciada (DIS), y con inexis- tencia de contacto táctil (CT)en los programas de fitness acuático, en el cycling y en la tonificación.

d) Diferencias muy marcadas en la orientación del instructor en cada actividad; la posición de enfrente (FC) es preeminente en la tonificación y singularmente en el fitness acuático cambia hacia la orientación de perfil (FP).

e) Diferencias en la transición del instructor; en el cycling es sentada (PFS), en el fitness acuático es de locomoción (LOC), y en el step y la tonificación se combina las posiciones bípedas desplazadas (PBD) y fija dorsal (PFD).

Tabla 4. Frecuencias de ocurrencias de las categorías de SOPROX-Fitness.

\begin{tabular}{llccccc}
\hline Criterio & Categoría & Fitness acuático & Cycling & Tonificación & Step & Total \\
\hline Grupo & Macro-grupo (MAC) & 75.63 & $\mathbf{8 7 . 4 5}$ & $\mathbf{8 7 . 9 8}$ & $\mathbf{9 9 . 0 9}$ & $\mathbf{6 6 . 1 8}$ \\
& Díada (DIA) & 17.82 & 10.51 & 10.86 & 0.59 & 9.95 \\
& Micro-grupo (MIC) & 6.55 & 2.04 & 1.16 & 0.32 & 23.87 \\
\hline \multirow{2}{*}{ Topología } & Periférica (P) & $\mathbf{9 9 . 8 3}$ & $\mathbf{9 9 . 0 5}$ & $\mathbf{8 8 . 5 9}$ & $\mathbf{9 9 . 8 3}$ & $\mathbf{9 6 . 8 4}$ \\
& Central (C) & 0.17 & 0.95 & 11.41 & 0.12 & 3.16 \\
\hline \multirow{2}{*}{ Interacción } & Integrada (IINT) & $\mathbf{9 9 . 0 3}$ & $\mathbf{9 8 . 8 4}$ & $\mathbf{9 5 . 7 2}$ & $\mathbf{9 9 . 8 2}$ & $\mathbf{9 8 . 3 5}$ \\
& Distanciada (DIS) & 0.97 & 1.16 & 2.50 & 0.18 & 1.20 \\
& Contacto Táctil (CT) & 0.00 & 0.00 & 1.78 & 0.00 & 0.45 \\
\hline
\end{tabular}




\begin{tabular}{llccccc}
\hline Criterio & Categoría & Fitness acuático & Cycling & Tonificación & Step & Total \\
\hline Orientación & De Frente Espejo (FE) & 58.97 & 98.44 & 16.50 & 71.14 & 61.26 \\
& De Frente Correspondiente (FC) & 1.77 & 0.47 & 59.05 & 28.19 & 22.37 \\
& De Frente Perfil (FP) & 23.01 & 0.54 & 7.00 & 0.67 & 7.80 \\
& Detrás (AT) & 3.16 & 0.00 & 1.09 & 0.00 & 1.07 \\
& En Medio (NM) & 0.00 & 0.00 & 8.85 & 0.00 & 2.21 \\
& Derecha (DR) & 3.58 & 0.00 & 2.73 & 0.00 & 1.58 \\
\hline \multirow{3}{*}{ Transición } & Izquierda (ESQ) & 9.51 & 0.55 & 4.78 & 0.00 & 3.71 \\
& P.B Desplazamiento (PBD) & 3.50 & 0.00 & 21.18 & 78.77 & 25.86 \\
& P.F. Bípeda (PFB) & 63.85 & 2.99 & 32.66 & 19.24 & 29.69 \\
& P.F. Sentado (PFS) & 2.71 & 97.01 & 7.25 & 0.79 & 26.94 \\
& P.F. Dorsal (PFD) & 0.00 & 0.00 & 19.36 & 0.00 & 4.84 \\
& P.F. Ventral (PFV) & 0.00 & 0.00 & 1.45 & 0.00 & 0.36 \\
& P.F. Lateral (PFL) & 0.00 & 0.00 & 1.23 & 0.00 & 0.31 \\
& Locomoción (LOC) & 29.94 & 0.00 & 16.87 & 1.20 & 12.00 \\
& En Soporte (SU) & 0.00 & 0.00 & 0.00 & 0.00 & 0.00 \\
\hline
\end{tabular}

Nota: resultados de las frecuencias expresados en porcentajes.

\section{Análisis inferencial}

Las relaciones significativas entre los gestos cinésicos y proxémicos en todas las actividades (ver tabla 5) revelan vínculos entre: a) RE-INF y entre IL-INT en todas las actividades analizadas, menos en step.

b) EMBN-INF, exceptuando en la tonificación.

c) INF-MAC y FEED-DIA exceptuando en la actividad de step.

Tabla 5. Contingencias significativas entre las categorías de actividades.

\begin{tabular}{|c|c|c|c|c|c|c|c|}
\hline \multicolumn{2}{|c|}{ Criterio } & \multicolumn{2}{|c|}{ Categoría } & \multicolumn{4}{|c|}{ Instructor } \\
\hline Fila & Columna & Fila & Columna & FITNESS acuático & FITNESS cycling & FITNESS tonificación & FITNESS step \\
\hline \multirow[t]{3}{*}{$\mathrm{DF}$} & DS & $\mathrm{RE}$ & INF & 12.37 & 8.08 & 7.78 & \\
\hline & & & FEED & 5.55 & & & \\
\hline & & IL & INT & 23.87 & 11.47 & 16.03 & \\
\hline \multirow[t]{5}{*}{$\mathrm{DM}$} & DS & DEI & FEED & 4.27 & & & \\
\hline & & EMBN & INF & 8.48 & 5.41 & & 5.58 \\
\hline & & EMBS & FEED & & 6.21 & & 5.82 \\
\hline & & & INT & 4.74 & 4.38 & & \\
\hline & & BAT & INT & 9.85 & 9.16 & & \\
\hline \multirow[t]{2}{*}{$\mathrm{DM}$} & DG & EMBN & MAC & 5.18 & 3.85 & & \\
\hline & & EMBS & DIA & & 4.10 & & \\
\hline \multirow[t]{3}{*}{ DS } & DG & INF & MAC & 16.28 & 13.51 & 8.38 & \\
\hline & & FEED & DIA & 11.75 & 9.35 & 7.59 & \\
\hline & & INT & DIA & 5.35 & 6.73 & & \\
\hline \multirow[t]{4}{*}{$\mathrm{DE}$} & DTR & $\mathrm{CE}$ & PFB & & & 8.01 & \\
\hline & & SE & LOC & 8.31 & & 10.42 & \\
\hline & & SE & PFB & & & & 10.38 \\
\hline & & $\mathrm{CE}$ & PBD & & & & 14.58 \\
\hline
\end{tabular}

Nota: resultados expresados en puntuaciones $z$ o residuos ajustados $(p<.001)$ 
Las relaciones significativas entre los gestos cinésicos y proxémicos internas a cada actividad (ver tabla 5) describen que:

a) En la tonificación el instructor usa un tipo de comunicación gestual simultánea al ejercicio (CE), en posición bípeda (PFB).

b) En el fitness acuático el instructor no realiza el ejercicio (SE) en el medio acuático pero se desplaza (LOC) continuamente.

c) En el step la participación del instructor se da simultánea a la demostración del ejercicio (CE) o sin esta demostración (SE) y en posición bípeda (PFB).

Después de este análisis estadístico descriptivo e inferencial se complementa los resultados obtenidos con un análisis de T-patterns que pueda aportarnos más referencias de cómo y cuando se producen los intercambios comunicativos entre las instructoras y los participantes en cada una de las actividades.

\section{Detección de patrones temporales (T-patterns)}

En las 12 sesiones observadas se registraron un total de 3242 comportamientos cinésicos y proxémicos con la siguiente distribución: fitness step $=957$; fitness tonificación $=591$; fitness acuático = 975; cycling $=719$. El análisis de los T-patterns evidencia vinculaciones temporales y secuenciales de la aparición de los gestos cinésicos y proxémicos, al obtener agrupaciones simultáneas de las categorías denominadas configuraciones conductuales. Estas agrupaciones están formadas por conjunto de de códigos concurrentes de cada actividad (Tabla 6), y representan configuraciones que se reparten así: 127 en el fitness step, 229 en la tonificación, 358 en el fitness acuático, y 130 en el cycling.

Tabla 6. Configuraciones de cada actividad y sus respetivas frecuencias absolutas, relativas y acumuladas.

\begin{tabular}{|c|c|c|c|c|}
\hline Actividades & Configuraciones & Frec. & $\%$ Total & $\%$ Acum. \\
\hline \multirow[t]{5}{*}{ Fitness Acuático } & 1a) RE,DEI,INF,CE,MAC,P,IINT,FE,PFB & 44 & 4.51 & 4.51 \\
\hline & $2^{\text {a) }}$ RE,EMBN,INF,SE,MAC,P,IINT,FP,LOC & 37 & 3.79 & 8.30 \\
\hline & 3a) RE,DEI,INF,SE,MAC,P,IINT,FE,PFB & 34 & 3.48 & 11.78 \\
\hline & 4a) RE,CIN,INF,SE,MAC,P,IINT,FE,PFB & 32 & 3.28 & 15.06 \\
\hline & 5a) RE,EMBN,INF,CE,MAC,P,IINT,FE,PFB & 25 & 2.56 & 17.62 \\
\hline \multirow[t]{5}{*}{ Cycling } & 1a) RE, CIN, INF, CE, MAC, P, IINT, FE, PFS & 115 & 15.99 & 15.99 \\
\hline & 2a) RE, EMBN, INF, CE, MAC, P, IINT, FE, PFS & 76 & 10.57 & 26.56 \\
\hline & 3a) RE, DEI, INF, CE, MAC, P, IINT, FE, PFS & 75 & 10.43 & 36.99 \\
\hline & 4a) RE,BAT,INF,CE,MAC,P,IINT,FE,PFS & 49 & 6.81 & 43.8 \\
\hline & 5a) RE,RIT,INF,CE,MAC,P,IINT,FE,PFS & 41 & 5.70 & 49.5 \\
\hline \multirow[t]{5}{*}{ Tonificación } & 1a) RE, DEI, INF, CE, MAC, P, IINT, FC, PBD & 31 & 5.24 & 5.24 \\
\hline & 2a) RE, EMBN, INF, CE, MAC, P, IINT, FC, PFD & 28 & 4.73 & 9.97 \\
\hline & 3a) RE, EMBN, INF, CE, MAC, P, IINT, FC, PBD & 26 & 4.39 & 14.36 \\
\hline & 4a) RE,CIN,INF,CE,MAC,P,IINT,FC,PFD & 18 & 3.04 & 17.4 \\
\hline & 5a) RE,EMBT,INF,CE,MAC,P,IINT,FC,PBD & 17 & 2.87 & 20.27 \\
\hline \multirow[t]{5}{*}{ Step } & 1a) RE, EMBT, INF, CE, MAC, P, IINT, FE, PBD & 130 & 13.58 & 13.58 \\
\hline & 2a) RE, DEI, INF, CE, MAC, P, IINT, FE, PBD & 129 & 13.47 & 27.05 \\
\hline & 3a) RE, DEI, INF, CE, MAC, P, IINT, FC, PBD & 87 & 9.09 & 36.14 \\
\hline & 4a) RE,EMBN,INF,CE,MAC,P,IINT,FE,PBD & 76 & 7.94 & 44.08 \\
\hline & 5a) RE,EMBT,INF,CE,MAC,P,IINT,FC,PBD & 54 & 5.64 & 49.72 \\
\hline
\end{tabular}

Estas configuraciones aparecen cronológicamente en cada actividad constituyendo patrones temporales (T-patterns) que se pueden representar en un diagrama de árbol o dendograma y que describe una tipología de conducta asociada a la actividad.

Para cada actividad, se presentaron los T-patterns con el mayor número de comportamientos combinados con tres ocurrencias mínimas y con un nivel de significación de $p<.05$.
Los T-patterns obtenidos en la activad de fitness acuático

La instructora (Fig.1) dibuja una acción con un regulador (RE-CIN) para informar (INF) sin demostración del ejercicio (SE) a todo el grupo (MAC) con interacción (INT) y con una posición de frente y de pie (FE-PFB); a continuación incorpora ritmo (RIT) y cambia la orientación de frente a los practicantes (FE) a la de estar de perfil a ellos (FP). 
Fig 1. Representación de los T-pattern de la actividad de fitness acuático.

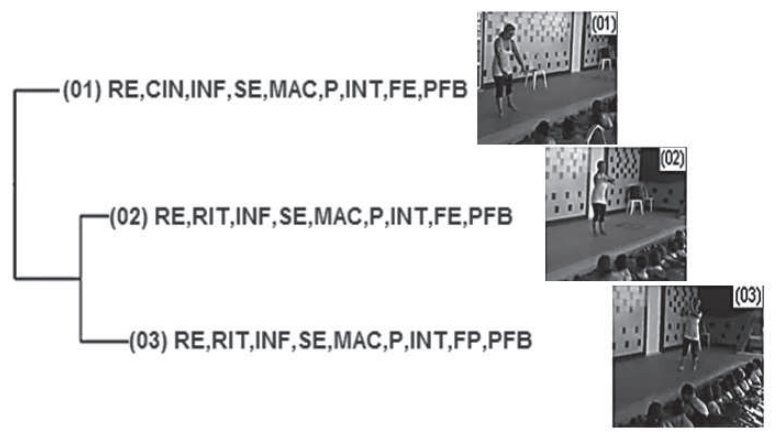

T-patterns obtenidos en la actividad de cycling

La instructora (Fig.2) representa una figura en el espacio con un regulador (RE-PIC) para informar (INF) mientras demuestra (CE) a todo el grupo (MAC) con interacción (INT) de frente y sentado en la bicicleta (FE-PFS); seguidamente (02-03-04) diversifica los gestos reguladores para dibujar un emblema numérico y para finalizar con un cinetográfico que imita una acción (ESP- EMBN CIN).

Fig 2. Representación del T-pattern de la actividad de cycling.

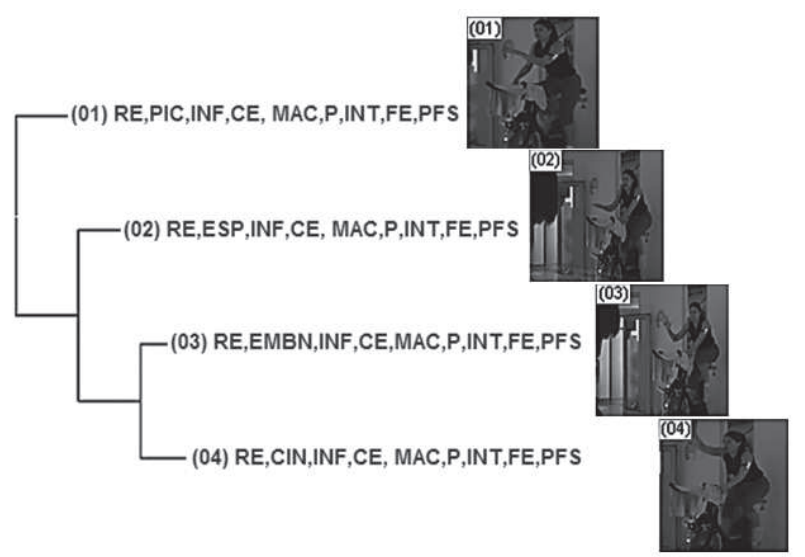

T-patterns obtenidos en la actividad de tonificación

La instructora (Fig.3) señala con un gesto regulador (REDEI-CE) para informar y participa con el grupo (INF-CEMAC) con interacción (INT) orientado como los practicantes (FC) y tumbado de espalda (PFD); seguidamente regula con un emblema numérico (EMBN) y lo alterna con un rítmico (RIT) e iconos establecidos (EMBS).
Fig 3. Representación de los T-pattern de la actividad de tonifcación.

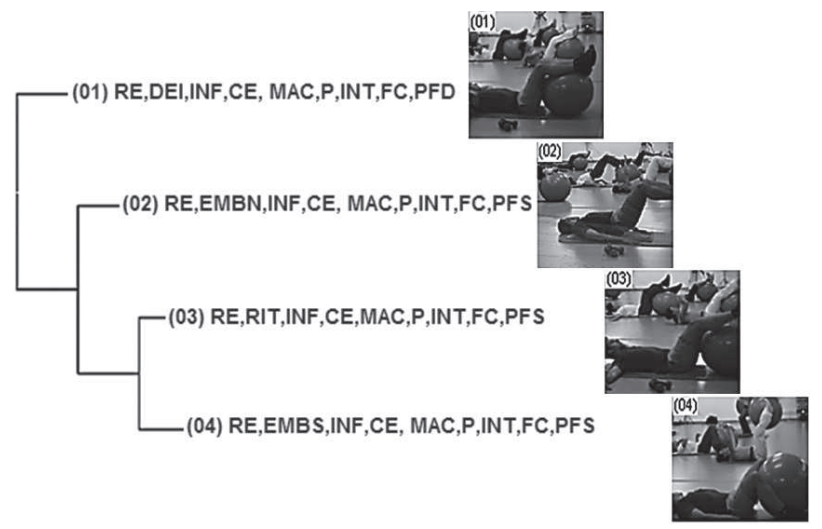

T-patterns obtenidos en la actividad de fitness step

La instructora (Fig.3) regula con emblemas de tiempo y deícticos (RE-EMBT-DEI), acompañándolo con emblemas sociales y numéricos (EMBS-EMBN), y con el objetivo de informar participando (INF-CE) a todo el grupo (MAC) en la periferia de la sala $(\mathrm{P})$ con interacción (INT), combinando la orientación de frente (FE) con la misma de los participantes $(\mathrm{FC})$ y de pie en desplazamiento (FBD).

Fig 4. Representación de los T-patterns de la actividad de fitness step.

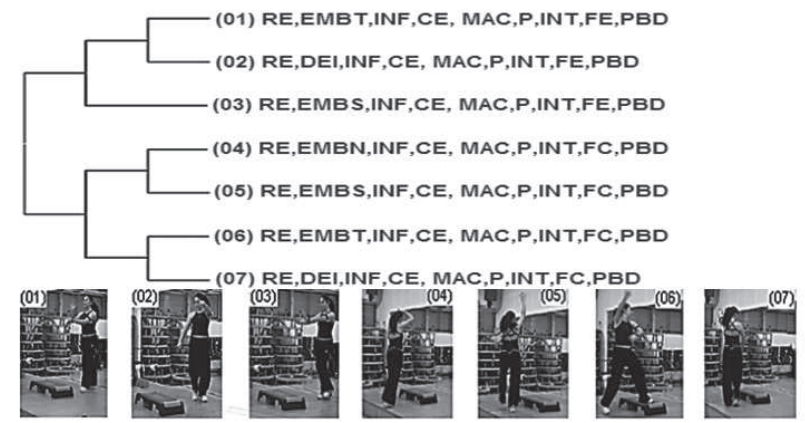

\section{Discusión y resultados}

Los resultados nos indican que la comunicación paraverbal de las instructoras de las actividades de fitness es diversificada (Goldin-Meadow, 2004), y está centrada en los comandos que aseguran el seguimiento de los ejercicios de la sesión por parte de los usuarios de cada programa que el monitor suele acompañar con demostraciones del ejercicio cargado de autoadaptadores. Así la información, distribuida a todo el grupo, se transmite con una regulación saturada de emblemas principalmente numéricos. Son escasas las situaciones en 
que se produce un proceso interactivo con los participantes y cuando existen es con gestos ilustradores y de forma individualizada o en díada. Podemos decir que el instructor es el principal protagonista por medio de unos modelos gestuales estandarizados y muy rutinizados tal como indican otros estudios (Alves et al., 2013; 2014).

Existe una mayor estabilidad conductual paraverbal entre las instructoras de fitness step y cycling al mostrar conductas cinésicas y proxémicas bastante comunes (tabla 6), entre el $49.50 \%$ y el $49.72 \%$ respectivamente de sus configuraciones registradas. Por otro lado, las instructoras de fitness acuático y tonificación desarrollan mayor diversificación paraverbal, con el $17.62 \%$ y el $20.27 \%$ respectivamente. Estas características comunes pueden justificarse por el estilo de enseńanza de mando directo (Francis y Seibert, 2000) que caracterizan la intervención de las instructoras de fitness en aulas de grupo y que condiciona su estilo comunicativo. Las variaciones conductuales paraverbales ocurren sobre todo en la morfología de los gestos, así como con diferentes ubicaciones del instructor con posturas corporales diversas (Alves et al., 2014; Castañer et al., 2013).

Este análisis fue complementado con los T-patterns, que permite la detección de patrones "ocultos" de comportamiento al suministrar pistas sobre la dinámica y secuencia de los gestos, su morfología, orientación y posición espacial. La complejidad de este tipo de análisis sugiere que su discusión sea efectuada por cada actividad.

En el fitness acuático la instructora está fuera del agua, y el T-pattern (Figura 1) identifica una regulación con desplazamientos de frente a los participantes a través de una secuencia de morfologías de gestos que imitan acciones, marcan un ritmo e informar sobre el ejercicio y su velocidad de ejecución. El hecho de que las instructoras están en el medio terrestre obliga a usar gestos que imitan acciones y marcan un ritmo para acompañar los practicantes. Por otro lado, las características de las piscinas y las malas condiciones acústicas de las piscinas dificulta la comunicación modelando una intervención paraverbal exigente y llena de gestualidad y desplazamientos en el proceso de enseńanza-aprendizaje.

En el cycling la instructora está sentado en la bicicleta, y el T-pattern (Figura 2) constata que la regulación del comportamiento de los practicantes se establece en una secuencia de morfología de gestos pictográficos que identifican distancias numeraciones e imitan acciones. La instructora modela con sus gestos lo que han de realizar los usuarios; una subida empinada que requiere la colocación de las manos más alejadas del manillar, o una imitación de la acción de pedalear en pie.

En la tonificación la instructora está en una posición fija de espaldas al practicante, y el T-pattern (Figura 3), constata una regulación deíctica, numérica y rítmica que puede acompańarse con un emblema social. La instructora señala el grupo muscular implicado e informa sobre el número de repeticio- nes y su velocidad de ejecución. Esta posición de espaldas dificulta su visualización, como demuestra el estudio sobre los comportamientos pedagógicos de los instructores de tonificación realizado por Franco, Rodrigues y Castañer (2008).

En el fitness step, tratándose de una actividad coreográfica, la instructora debe representar primero el movimiento con desplazamientos derivados de los propios ejercicios de frente a sus alumnos para identificar la habilidad motora. Seguidamente regulan el comportamiento a través de emblemas sociales y cambiando su orientación quedando de espalda a los practicantes. El T-pattern detectado se justifica por las características del proceso de enseñanza de las habilidades implicadas en esta actividad.

\section{Conclusiones}

La metodología observacional (Anguera, 2009) y los sistemas de observación adaptados para el estudio del comportamiento cinésico y proxémico (Castañer et al., 2013) en el contexto del fitness (Alves et al., 2014) se revelaron eficaces para constatar el comportamiento comunicativo de los instructores a través de la identificación de los diferentes patrones temporales de comportamiento (T-patterns) en un contexto natural de observación (Magnusson, 2006). El análisis de estos patrones han contribuido significativamente a la optimización de la comunicación de los profesionales de fitness. Siendo este un estudio exploratorio, consideramos importante proseguir con la realización de nuevas investigaciones futuras que puedan replicar estos resultados referidos a las diversas actividades de fitness ya que, como refieren (Castańer et al., 2013), siendo la comunicación una parte importante del proceso de enseñanza-aprendizaje, una de las formas de mejorar ese proceso pasa por tomar atención a la forma de optimizarla a lo largo de la experiencia del profesional (Mc Neill, 2005).

La importancia de la comunicación paraverbal en este contexto es fundamental, permitiendo una mayor rapidez y claridad en la transmisión de la información y evitando esfuerzos excesivos en la voz que pueden ocasionarle lesiones en las cuerdas vocales teniendo unos efectos profilácticos (Long, Williford, Olson, y Wolfe, 1998).

Esta inmediatez crea una dependencia de la instructora hacia la repetición y la automatización de movimientos que le resta capacidad interactiva con sus alumnos y aumenta la dependencia con el grupo masivo; no ofreciendo posibilidades de interacciones participativas de los participantes, ni una corrección y trato individualizado (Roth, 1999).

\section{Aplicaciones prácticas}

La mejora de las estrategias didácticas podría ofrecer una alternativa a esta dependencia gestual de los monitores en aras al incremento de su capacidad comunicativa interactiva de 
mayor satisfacción profesional. Sería necesario innovar estrategias comunicativas y psicológicas que desarrollen de manera eficaz el discurso de los futuros instructores para aumentar su capacidad interactiva y liberarlos de una única subordinación a la gestualidad de tipo regulador.

La sensibilización sobre la importancia del dominio de la comunicación paraverbal debería ser entrenada en los procesos de enseńanza-aprendizaje (Berliner, 2001), y tendría que estar potenciada dentro de la mejora comunicativa y profesional de los instructores para beneficiar el clima del aula, la motivación y adhesión de los practicantes de fitness (Castañer et al. 2014; Saüch y Castañer, 2014).
Agradecimientos: Este articulo se ha realizado con el apoyo y financiación de: Grup de recerca i innovació en dissenys (GRID). Tecnologia $i$ aplicació multimedia $i$ digital als dissenys observacionals. Grupo Consolidado de la Generalitat de Catalunya [Referencia: 2009 SGR 829]. Asimismo, ha recibido el apoyo del Proyecto $\mathrm{I}+\mathrm{D}+\mathrm{I}$ : Observación de la interacción en deporte y actividad fisica: Avances técnicos y metodológicos en registros automatizados cualitativos-cuantitativos. Secretaria de Estado de Investigación, Desarrollo e Innovación del Ministerio de Economía y Competitividad [Referencia: DEP2012-32124].

\section{Referencias}

1. Alves, S., Rodrigues, J., Castañer, M., Camerino, O., Sequeira, P., Carvalhinho, L., Simóes, V., Franco, S. (2013). Sistema de Observaçáo da Comunicação Proxémica do Instrutor de Fitness (SOPROX-Fitness): Desenvolvimento, Validação e Estudo Piloto. Revista Iberoamericana de Psicología del Ejercicio y el Deporte, 8(2), 281-299.

2. Alves, S., Rodrigues, J., Castañer, M., Camerino, O., Sequeira, P., Carvalhinho, L., Simões, V., Franco, S. (2014). Validação e Desenvolvimento de um Sistema de Observação da Comunicação Cinésica do Instrutor de Fitness Motricidade, 10(1), 77-87.

3. Anguera, T. (2009). Methodological observation in sport: Current situation and challenges for the next future. Motricidade 5(3), 15-25.

4. Anguera, M. T., Blanco-Villaseñor, Á., y Losada, J. L. (2001). Diseńos Observacionales, Cuestión Clave en el Proceso de la Metodología Observacional. Metodología de las Ciencias del Comportamiento, 3(2), 135-160.

5. Anguera, M. T., Blanco-Villaseñor, A., Hernández-Mendo, A., y Losada, J. L. (2011). Diseños observacionales: ajuste y aplicación en psicología del deporte. Cuadernos de Psicología del Deporte, 11(2),63-76

6. Anguera, M.T., Camerino, O., Castañer, M. y Sánchez-Algarra, P. (2014). Mixed methods en actividad física y deporte. Revista de Psicología del Deporte, 23(1), 123-130.

7. Anguera, M.T. y Hernández-Mendo, A. (2014). Metodología observacional y psicología del deporte: Estado de la cuestión. Revista de Psicología del Deporte, 23(1), 103-109.

8. Bakeman, R., y Quera, V. (1995). Analyzing interaction: Sequential analysis with SDIS and GSEQ. Cambridge: Cambridge University Press.

9. Battista, R., Foster, C., Andrew, J., Wright, G., Lucia, A., y Porcari, J. (2008). Physiologic responses during indoor cycling. Journal of Strength and Conditioning Research, 22(4), 1236-1241.

10. Berry, M., Cline, C., Berry, C., y Davis, M. (1992) A comparison between two forms of aerobic dance and treadmill running. Medicine and Sxience in Sports and Exercise 6(4), 415-425.

11. Berliner, D. C. (1994). Teacher Expertise. En A. Pollard y J. Bourne (Eds.), Teaching and learning in the primary school (pp. 73-79). London: Routledge.

12. Brewer, C. J., y Jones, R. L. (2002). A Five-Stage Process for Establishing Contextually Valid Systematic Observation Instruments: The Case of Rugby Union. The Sport Psychologist, 16(2), 138-159.

13. Camerino, O., Castañer, M., y Anguera, M. T. (Eds.). (2012). Mixed Methods Research in the Movement Sciences: Cases in Sport, Physical Education and Dance. London: Routledge.

14. Cartmill, E. A., Goldin-Meadow, S., y Beilock, S. L. (2012). A word in the hand: Human gesture links representations to actions. Philosophical Transactions of the Royal Society, 367(1585), 129-143.

15. Castañer, M. (2009). SOCOP, sistema de observación para la optimi- zación de la comunicación paraverbal del docente. Temps d'Educació, 36, 231-246.

16. Castañer, M., Torrents, C., Anguera, M.T., Dinušová, M., y Jonsson, G.K. (2009). Identifying and analyzing motor skill responses in body movement and dance. Behavior Research Methods, 41(3), 857-867.

17. Castañer, M., Camerino, O., Anguera, M. T., y Jonsson, G. K. (2010). Observing the paraverbal communicative style of expert and novice PE teachers by means of SOCOP: a sequential analysis. Procedia - Social and Behavioral Sciences, 2(2), 5162-5167.

18. Castañer, M., Franco, S., Rodrigues, J., y Miguel, C. (2012). Optimizing verbal and nonverbal communication in PE teachers, instructors and sport coaches. En O. Camerino, M. Castañer y M. T. Anguera (Eds.), Mixed Methods Research in the Movement Sciences: Cases in Sport, Physical Education and Dance (pp.202-214). London: Routledge.

19. Castañer, M., Camerino, O., Anguera, M. T., y Jonsson, G. K. (2013). Kinesics and proxemics communication of expert and novice PE teachers. Quality y Quantity, 47(4), 1813-1829.

20. Castañer, M., Camerino, O., y Saüch, G., (2014). La comunicació proxèmica en l'acció docent dels pedagogs-animadors de les activitats físiques per a la gent gran. Temps d'educació, 46, 167-184.

21. Cohen, J. (1960). A coefficient of agreement for nominal scales. Educational and Psychological Measurement, 20, 37-46.

22. Francis, L. L., y Seibert, R. J. (2000). Teaching a Group Exercise Class. En ACE (Ed.), Group Fitness Instructor Manual (pp. 178-204). San Diego: American Council on Exercise.

23. Franco, S., Rodrigues, J., y Castañer, M. (2008). Comportamento pedagógico dos personal trainers de aulas de grupo de fitness de localizada. Fitness y Performance Journal, 7(4), 251-263.

24. Franco, S., y Santos, R. (1999). A Essência da Ginástica Aeróbica. Rio Maior: Ediçōes ESDRM.

25. Gabín, B., Camerino, O., Anguera, M. T., y Castañer, M. (2012). Lince: multiplatform sport analysis software Procedia - Social and Behavioral Sciences, 46, $4692-4694$

26. Goldin-Meadow, S. (2004). Gesture's role in the learning process. Theory into Practice, 43(4), 314-321. doi: 10.1207/s15430421tip4304_10

27. Harriss, D. J., y Atkinson, G. (2009). International Journal of Sports Medicine - Ethical Standards in Sport and Exercise Science Research. International Journal of Sports Medicine, 30(10), 701-702.

28. Harriss, D. J., y Atkinson, G. (2011). Update - Ethical Standards in Sport and Exercise Science Research. International Journal of Sports Medicine, 30(10), 701-702.

29. Hernández-Mendo, A., Castellano, J., Camerino, O., Jonsson, G.K., Blanco-Villaseñor, A., Lopes, A. y Anguera, M.T. (2014). Programas informáticos de registro, control de calidad del dato, y análisis de datos. Revista de Psicología del Deporte, 23(1), 111-121. 
30. Hostetter, A. B. (2011). When do gestures communicate? A metaanalysis. Psychological Bulletin, 137(2), 297-315.

31. Jonsson, G., Anguera, M., Sánchez-Algarra, P., Oliveira, C., Campaniço, J., Castañer, M., Torrents, C., Dinusova, M., Chaverri, J., Camerino, O., y Magnusson, M. (2010). Application of T-Pattern Detection and Analysis in Sports Research. The Open Sport Sciences Journal, 3, 95-104.

32. Kendon, A. (2004). Gesture: Visible Action as Utterance. New York, NY: Cambridge University Press.

33. Kennedy, C. A., y Yoke, M. M. (2004). Methods of Group Exercise Instruction. IL, Champaign: Human Kinetics.

34. Kennedy, C. W., y Camden, C. (1983). Interruptions and nonverbal gender differences. Journal of Nonverbal Behavior, 8(2), 91-108.

35. Knapp, M. L., y Hall, J. A. (2010). Nonverbal Communication in Human Interaction (7th ed.). Wadsworth: Thomas Learning.

36. Long, J., Williford, H. N., Olson, M,S. y Wolfe, V. (1998). Voice problems and risk factors among aerobics instructors. Journal of Voice, 12(2), 197-207.

37. Magnusson, M. S. (2000). Discovering hidden time patterns in behavior: T-patterns and their detection. Behaviour Research Methods, Instruments \& Computers, 32(1), 93-110.

38. Magnusson, M. S. (2005). Understanding social interaction: discovering hidden structure with model and algorithms. En L. Anolli, S. J. Duncan, M. S. Magnusson y G. Riva (Eds.), The Hidden Structure of Interaction: From Neurons to Culture Patterns (pp. 3-22). Amsterdam: IOS Press.

39. Magnusson, M.S. (2006) Structure and Communication in Interaction. En G. Riva, M.T. Anguera, B.K. Wiederhold \& F. Mantovani
(Eds.), From Communication to Presence: Cognition, Emotions and Culture Towards the Ultimate Communicative Experience (pp. 127-146). Amsterdam: IOS Press

40. Malek, M. H., Nalbone, D. P., Berger, D. E., y Coburn, J. W. (2002). Importance of health sciences education for personal fitness trainers. Journal of Strength \& Conditioning Research, 16(1), 19-24.

41. McNeill, D. (2005). Gesture and Thought. Chicago: University of Chicago Press.

42. Mirbod, S. M., Lanphere, C., Fujita, S., Komura, Y., Inaba, R., y Iwata, H. (1994). Noise in aerobic facilities. Industrial Health, 32, 49-55.

43. Moreno, J. A., y Martínez, A. (2006). Importancia de la Teoría de la Autodeterminación en la práctica físico-deportiva: Fundamentos e implicaciones prácticas. Cuadernos de Psicología del Deporte, 6(2), 39-54.

44. Moreno, J.A., Cervelló, E., Marcos, P.J., y Martín, E.H., (2010). Importancia de la valoración del comportamiento autónomo del practicante para el disfrute en programas de ejercicio físico acuático. Cuadernos de Psicología del Deporte, 10(1), 57-70.

45. Roth, W. M (2001). Gestures: Their role in teaching and learning. Review of Educational Research, 71(3), 365-392.

46. Saüch, G., y Castañer, M. (2014). Observación de patrones motrices generados por los programas de actividad física para la tercera edad y la percepción de sus usuarios. Psicología del Deporte, 23(1), 181-190.

47. Simôes, V., Franco, S., y Rodrigues, J. (2009). Estudo do feedback pedagógico em personaltrainers de ginástica localizada com diferentes níveis de experiência profissional. Fitness \& Performance, 8(3), 174-182.

48. Yarenchuk, K. L., y Kaczor, J. C. (1999). Noise levels in the health club setting. Ear Nose \& Throat Journal, 78, 54-57. 